\title{
Basal perturbations under ice streams: form drag and surface expression
}

\author{
Christian SCHOOF ${ }^{*}$ \\ Mathematical Institute, Oxford University, 24-29 St Giles', Oxford OX1 3LB, England \\ E-mail: cschoof@eos.ubc.ca
}

\begin{abstract}
Glassical sliding theories consider ice sliding over obstacles which are much shorter than the thickness of overlying ice. Here we present a theory which considers "form drag" generated under ice streams by large obstacles such as subglacial bedforms, which may have lengths comparable to ice thickness. We also investigate how perturbations at the surface of an ice stream can be generated by such bedforms, and develop a mathematical framework for separating the effects of such local (kilometre-scale) variations in ice flow from the bulk flow of the ice stream.
\end{abstract}

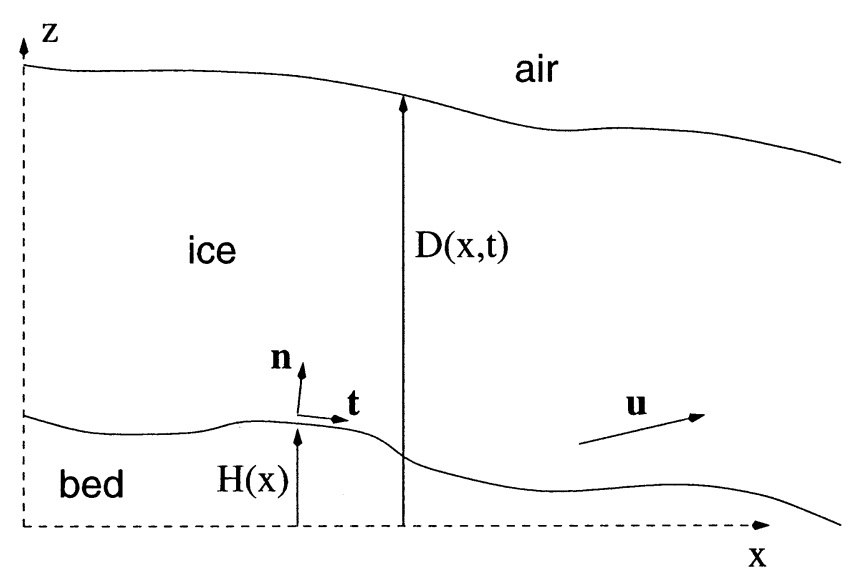

Fig. 1. Geometry of ice-flow problem.

\section{INTRODUGTION}

Traditionally, analytical studies of glacier and ice-sheet flow have considered length scales in the downstream direction which are either much shorter or longer than the thickness of ice. The former corresponds to classical models of basal sliding (e.g. Nye, 1969, and particularly Fowler, 1979, 1981), while the latter leads to the shallow-ice approximation (e.g. Hutter, 1983). A consistent model which deals with ice flow over basal undulations whose wavelengths are comparable with ice thickness is, however, desirable as it could elucidate how large obstacles such as drumlins and other glacial bedforms generate "form drag".

Here we present a two-dimensional model for rapid ice flow over such large-wavelength obstacles (i.e. of lengths comparable to ice thickness). We restrict ourselves to the

\footnotetext{
* Present address: Department of Earth and Ocean Sciences, University of British Columbia, 6339 Stores Road, Vancouver, British Columbia V6T 1Z4, Canada.
}

case where these obstacles play a significant role in the force balance of the ice flow, analogous to classical basal sliding. Given a stress tensor $\sigma$ and a bed $z=H(x)$ with normal $\mathbf{n}$ and tangent $\mathbf{t}$ (cf. Fig. 1), the force exerted by a section of bed on the body of ice is

$$
-\left.\int \sigma \mathbf{n}\right|_{\mathbf{z}=\mathbf{H}} \mathrm{d} \mathbf{s}=-\left.\int \mathbf{n} \cdot \sigma \mathbf{n}\right|_{\mathbf{z}=\mathbf{H}} \mathbf{n} \mathrm{d} \mathbf{s}-\left.\int \mathbf{t} \cdot \sigma \mathbf{n}\right|_{\mathbf{z}=\mathbf{H}} \mathbf{t} \mathrm{d} \mathbf{s}
$$

where the integral is taken over the section of bed in question and $\mathrm{d} s$ denotes an element of arc of the bed. In classical hard-bed sliding, local basal shear stress $\left.\mathbf{t} \cdot \sigma \mathbf{n}\right|_{z=H}$ is usually assumed to be negligible due to the presence of a continuous regelation film. A thin weak layer of deforming sediment (e.g. Engelhardt and Kamb, 1998) under an ice stream could have a similar effect; moreover, it might suppress small-scale roughness generated by small obstacles such as clasts, as these would be sheared along with the till matrix. It is, however, conceivable that sufficiently large obstacles such as drumlins may contribute to force balance in the downstream direction through the first term on the righthand side above, which we have referred to as "form drag". The theory constructed in sections 2-5 assumes that form drag is significant, and the scalings introduced in section 4 reflect this. Consequently, our theory is an extension of classical basal sliding theory to finite ice depth. We do not, however, ignore the possibility of smallscale roughness or local basal shear stress due to sediment deformation, but merely assume that basal "friction" due to these terms does not dominate over the effect of large-scale bed topography in determining flow velocities. This will be the case for a given bed topography if basal "friction" is sufficiently small, or, for a given local sliding law, if bed topography has sufficient amplitude. An analogous approach was previously taken in classical sliding theory by Morland (1976b) and Fowler (1981).

Certain restrictions on mean bed and surface slope (cf. sections 4 and 5) do, however, have to be applied to our theory; essentially the mean surface slope of the ice body has to be much smaller than the local bed roughness slope, which in 


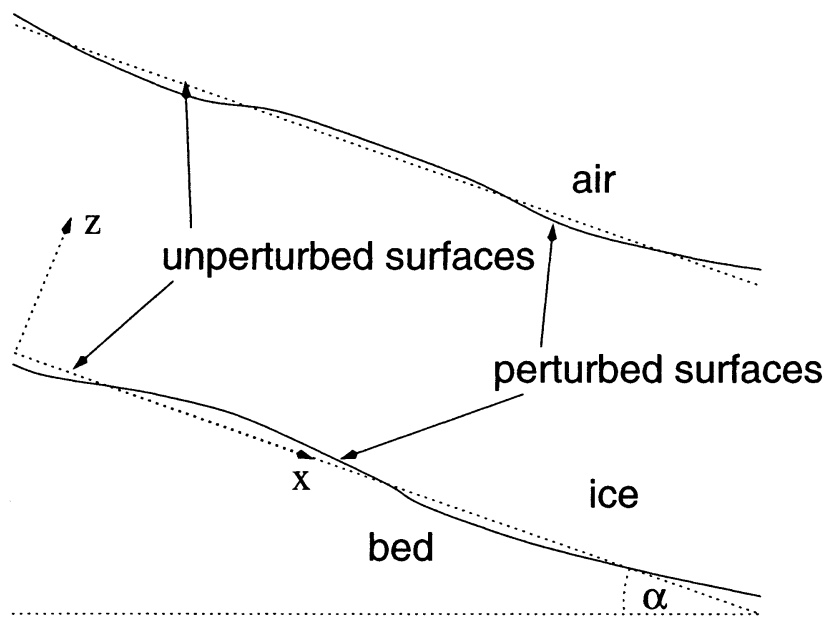

Fig. 2. Problem considered by Gudmundsson and others (1998).

turn is small compared with unity. This would obviously apply to drumlin-type basal topography under an ice stream; consequently most of the subsequent discussion refers to ice streams. While there is no unambiguous evidence that drumlins or other bedforms exist under present-day ice streams, geological evidence suggests that rapid sliding did occur over drumlinized terrain, for instance in the Puget Sound area (U.S.A.) during the last Ice Age (e.g. Brown and others, 1987).

Morland (1976a) was the first author to try to include the notion of finite ice depth in a theory of basal sliding. However, his solution of the flow equations does rely on a half-space (infinite depth) geometry, and he does not deal with perturbations caused at the glacier surface by bumps on the bed. Moreover, the ice-flow geometry considered by Morland is an inclined slab, whereas we consider a general ice-stream geometry and show explicitly how to separate the local flow problem over basal topography from the problem of the bulk flow of the ice stream; this may be seen as analogous to the asymptotic matching procedure of Fowler (1979, 1981), which relates the local basal sliding problem to the problem of bulk glacier flow. In the present case, however, the separation of the two flow problems is considerably more involved since they cannot be separated geometrically into a boundary layer and an outer flow. Instead, a more complicated multiplescales approach must be used.

The assumption of significant form drag sets this analysis apart from those of Gudmundsson and others (1998), who studied the effect of basal perturbations on ice-stream surfaces, of Morland (2000), who considered basal undulations under ice sheets, and those of other authors who have considered the effect of basal perturbations on finite-depth ice flow (e.g. Hutter and others, 1981; Hutter, 1983; Reeh, 1987). All of these authors consider perturbations to a basic shearing flow, where form drag is a higher-order correction, whereas our analysis assumes that form drag is of leading order. Indeed, our model may be applied to the case where there is no small-scale roughness and hence no "sliding law" in the classical sense, whereas Gudmundsson and others (1998) and Morland (2000) require a sliding law to be prescribed. However, as both Gudmundsson and others (1998) and the present paper consider rapid ice flow over basal topography, some of the results obtained are similar. As we point out in section 3, the two models basically differ in assumptions about the size of basal obstacles and of basal shear stresses.

A two-dimensional model, by its very nature, has to neglect lateral shear (e.g. Whillans and Van der Veen, 1997).
However, as lateral shear is unlikely to vary over distances comparable with ice thickness (since ice streams are much wider than thick), this neglect is tantamount to ignoring a constant term in the local force balance, or equivalently, to overestimating the driving stress.

\section{MODEL}

The geometry of the problem is illustrated in Figure 1. We consider the flow of Newtonian viscous ice over a prescribed bed $z=H(x)$, while $z=D(x, t)$ denotes the upper surface of the ice. Given viscosity $\eta$, density $\rho$ and acceleration due to gravity $g$, and denoting the velocity field by $\mathbf{u}=(u, w)$, we obtain the usual slow-flow equations

$$
\begin{gathered}
\eta \nabla^{2} \mathbf{u}-\nabla p-\rho g \mathbf{k}=0, \\
\nabla \cdot \mathbf{u}=0,
\end{gathered}
$$

where $\mathbf{k}$ is the $z$-unit vector. At the surface $z=D$, we prescribe vanishing shear and normal stress,

$$
\begin{aligned}
& \frac{\eta}{1+\left(\frac{\partial D}{\partial x}\right)^{2}} \\
& {\left[\left(1-\left(\frac{\partial D}{\partial x}\right)^{2}\right)\left(\frac{\partial u}{\partial z}+\frac{\partial w}{\partial x}\right)+2 \frac{\partial D}{\partial x}\left(\frac{\partial w}{\partial z}-\frac{\partial u}{\partial x}\right)\right]=0,}
\end{aligned}
$$

$p-\frac{2 \eta}{1+\left(\frac{\partial D}{\partial x}\right)^{2}}\left[\frac{\partial w}{\partial z}+\left(\frac{\partial D}{\partial x}\right)^{2} \frac{\partial u}{\partial x}-\frac{\partial D}{\partial x}\left(\frac{\partial w}{\partial x}+\frac{\partial u}{\partial z}\right)\right]=0$

while the evolution of $D(x, t)$ is governed by a kinematic boundary condition (where accumulation is ignored)

$$
\frac{\partial D}{\partial t}+u \frac{\partial D}{\partial x}=w \quad \text { on } z=D .
$$

At the base of the ice stream $z=H$, we suppose that there may be an applied shear stress $\tau_{\mathrm{b}}\left(u_{\mathrm{b}}, x\right)$ which is a function of basal sliding velocity $u_{\mathrm{b}}$ and position $x$,

$$
\begin{aligned}
\frac{\eta}{1+\left(\frac{\partial H}{\partial x}\right)^{2}}\left[\left(1-\left(\frac{\partial H}{\partial x}\right)^{2}\right)\left(\frac{\partial u}{\partial z}+\frac{\partial w}{\partial x}\right)+2 \frac{\partial H}{\partial x}\left(\frac{\partial w}{\partial z}-\frac{\partial u}{\partial x}\right)\right] \\
=\tau_{b}\left(u_{b}, x\right) \\
u_{\mathrm{b}}=u\left(1+\left(\frac{\partial H}{\partial x}\right)^{2}\right)^{1 / 2}
\end{aligned}
$$

$\tau_{\mathrm{b}}$ may arise if there is some microscale roughness or if there is a thin deforming sediment layer with sufficient shear strength. For a bed with no small-scale resistance, we simply put $\tau_{\mathrm{b}}=0$. Lastly, we have a kinematic boundary condition analogous to Equation (5) where basal melt is ignored,

$$
w=u \frac{\partial H}{\partial x} \quad \text { on } z=H \text {. }
$$

\section{GUDMUNDSSON'S MODEL}

Gudmundsson and others (1998) assume that both $z=H$ and $z=D$ are straight, parallel lines inclined at some angle $\alpha$ to the horizontal, and then perturb them slightly (see Fig. 2 ; in fact, it is convenient to realign the coordinate system with these straight lines). A linearization of the equations in the previous section (with a particular choice of sliding 


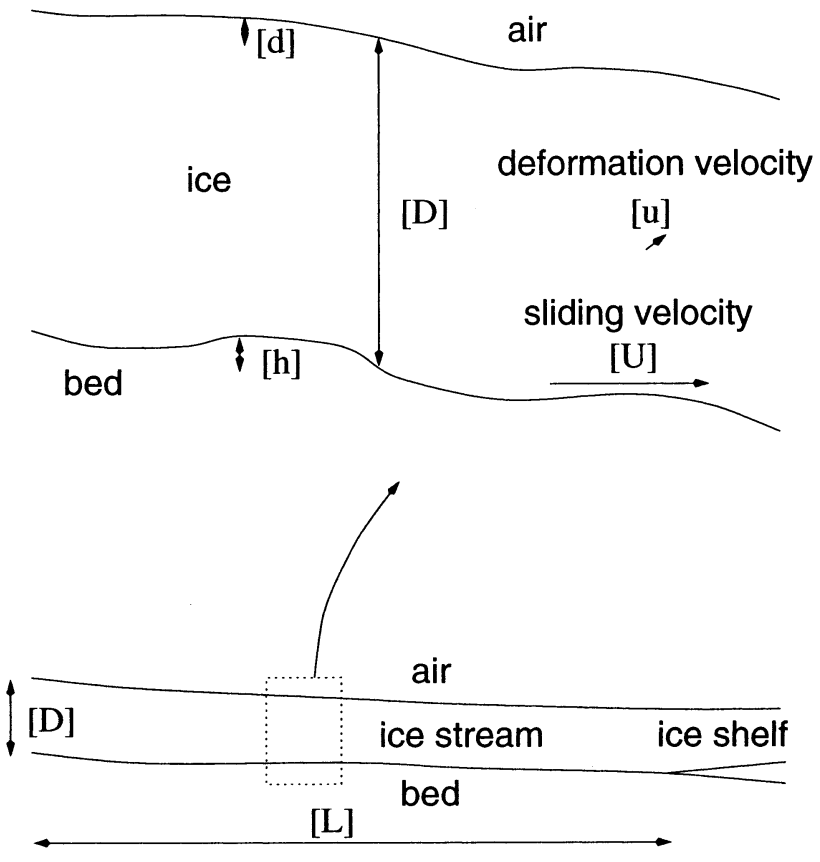

Fig. 3. Scales for the ice-stream flow problem.

law $\tau_{\mathrm{b}}$ ) is then performed on the basis that the perturbations introduced are small.

A crucial point, however, is that small geometrical perturbations may in fact introduce $O(1)$ or even large perturbations into the stress field when sliding is rapid. To understand this, consider a bed perturbation of size $h$ and wavelength $D$ (scales for bed bump height and ice thickness, respectively) in Equation (8). Vertical velocity is then perturbed by an amount $w \sim u_{\mathrm{b}} h / D$ by such a bump. If this perturbation is of $O(1)$ compared with the unperturbed shearing velocity in the ice $u_{\mathrm{s}}$, then a linearization of boundary condition (7) may fail, depending on the form of $\tau_{\mathrm{b}}$ in Equation (8). It is easy to see that, if sliding is rapid in the sense that $u_{\mathrm{b}} / u_{\mathrm{s}} \gg$ $1, w \sim u_{\mathrm{s}}$ occurs for fairly subdued bumps, namely, when $h / D \sim u_{\mathrm{s}} / u_{\mathrm{b}}$. Gudmundsson and others (1998) estimate $u_{\mathrm{b}} / u_{\mathrm{s}}$ at around 5000 for a typical ice stream, so $w \sim u_{\mathrm{s}}$ corresponds to $h \approx 0.2 \mathrm{~m}$ when $D=1000 \mathrm{~m}$. If $w$ is large compared with $u_{\mathrm{s}}$ - and the previous argument indicates that this may in fact be the case when $h$ is still quite small - then we may see terms in Equation (7) become important which are neglected, regardless of the choice of $\tau_{\mathrm{b}}$, in Gudmundsson and others' linearization. These terms include the "normal stress" effects which lead to form drag in classical basal sliding theory, and the rest of this paper is concerned with the situation where they are important.

\section{NON-DIMENSIONALIZATION}

We define the following scales (see Fig. 3): $[D]$ for the mean thickness of the ice stream (and also for the mean variation in bed elevation over the length of the ice stream), $[L]$ for the length of the ice stream, $[U]$ for a typical basal sliding velocity and $[u]$ for typical internal deformation velocities (note that $[U]$ and $[u]$ are distinct and may be asymptotically different). Moreover, we suppose that there are bumps of wavelength $\sim[D]$ and amplitude $[h]$ on the bed, and corresponding perturbations of height $[d]$ to the ice-stream surface. $[\tau]$ will denote a typical deviatoric stress, while $\left[\tau_{\mathrm{d}}\right]$ is a scale for the driving stress, distinct from $[\tau]$. Note that, because we scale the change in bed elevation $H$ over the length of the ice stream $L$ with $[D]$, these scalings would not apply to a steep glacier geometry.

As in shallow-ice theory, the mean surface slope of the ice stream is assumed to be small,

$$
\epsilon \doteq \frac{[D]}{[L]} \ll 1 .
$$

There are then two important asymptotically different horizontal length scales, $[D]$ and $[L]$. We are mostly interested in the local flow problem on the short length scale $[D]$, but the driving stress arises only because there is a mean decrease in surface elevation in the downstream direction over the length of the ice stream. One cannot therefore ignore variations on the long scale entirely, and this suggests multiply scaled horizontal coordinates and associated time variables

$$
\begin{aligned}
& x=[L] X^{\star}=[D] x^{\star}, \\
& t=\frac{[L] T^{\star}}{[U]}=\frac{[D] t^{\star}}{[U]} .
\end{aligned}
$$

$\left(x^{\star}, t^{\star}\right)$ and $\left(X^{\star}, T^{\star}\right)$ are, of course, not independent, as $\left(X^{\star}, T^{\star}\right)=\epsilon\left(x^{\star}, t^{\star}\right)$. In the limit $\epsilon \ll 1$ one can, however, treat them as independent in the context of a multiple-scales expansion (e.g. Holmes, 1995). The "inner" variables $\left(x^{\star}, t^{\star}\right)$ can be thought of as describing local variations, whereas the "outer" ones $\left(X^{\star}, T^{\star}\right)$ describe the bulk behaviour of the ice stream. The appropriate transformation of derivatives is then

$$
\begin{gathered}
\frac{\partial}{\partial x}=\frac{1}{[D]}\left(\frac{\partial}{\partial x^{\star}}+\epsilon \frac{\partial}{\partial X^{\star}}\right), \\
\frac{\partial}{\partial t}=\frac{[U]}{[D]}\left(\frac{\partial}{\partial t^{\star}}+\epsilon \frac{\partial}{\partial T^{\star}}\right) .
\end{gathered}
$$

In his account of classical sliding, Fowler (1981) separated bed elevation into a smoothed bed and a local roughness component. Following his example, we define a smoothed bed elevation as a running average:

$$
[D] H^{\star}\left(X^{\star}\right)=\frac{1}{2 L_{\mathrm{M}}} \int_{-L_{\mathrm{M}}}^{L_{\mathrm{M}}} H(x+\xi) \mathrm{d} \xi,
$$

where $[D] \ll L_{M} \ll[L]$ so that the smoothed bed thus defined depends only on the outer variable $X^{\star}$. Implicit in this definition is the assumption that basal topography occurs on asymptotically distinct length scales $[D]$ and $[L]$ with insignificant topography of intermediate wavelengths, which makes the smoothing above unique (cf. also Fowler, 1981). In practice, one might choose for $L_{\mathrm{M}}$ the geometric mean of $[D]$ and $[L], L_{\mathrm{M}}=([D][L])^{1 / 2} \approx 20 \mathrm{~km}$ for a typical ice stream. It is expedient to apply a similar smoothing procedure to surface elevation and velocity, thus

$$
\begin{gathered}
{[D] D^{\star}\left(X^{\star}, t^{\star}, T^{\star}\right)=\frac{1}{2 L_{\mathrm{M}}} \int_{-L_{\mathrm{M}}}^{L_{\mathrm{M}}} D(x+\xi, t) \mathrm{d} \xi} \\
{[U] U^{\star}\left(X^{\star}, t^{\star}, T^{\star}\right)=\left.\frac{1}{2 L_{\mathrm{M}}} \int_{-L_{\mathrm{M}}}^{L_{\mathrm{M}}} u(x+\xi, t)\right|_{z=H} \mathrm{~d} \xi .}
\end{gathered}
$$


These decompositions allow the following dimensionless variables to be defined in addition to Equations (11) and (12):

$$
\begin{aligned}
z= & {[D] z^{\star}, } \\
\mathbf{u}= & \mathbf{i}[U] U^{\star}\left(X^{\star}, t^{\star}, T^{\star}\right)+\mathbf{k} \epsilon[U]\left(U^{\star} \frac{\partial H^{\star}}{\partial X^{\star}}-\left(z^{\star}-H^{\star}\right) \frac{\partial U^{\star}}{\partial X^{\star}}\right) \\
& +[u] \mathbf{u}^{\star}\left(x^{\star}, z^{\star}, t^{\star}, X^{\star}, T^{\star}\right), \\
H= & {[D] H^{\star}\left(X^{\star}\right)+[h] h^{\star}\left(x^{\star}, X^{\star}\right), } \\
D= & {[D] D^{\star}\left(X^{\star}, t^{\star}, T^{\star}\right)+[d] d^{\star}\left(x^{\star}, t^{\star}, X^{\star}, T^{\star}\right), } \\
\tau_{\mathrm{b}}= & {\left[\tau_{\mathrm{d}}\right] \tau_{\mathrm{b}}^{\star}, \quad u_{\mathrm{b}}=[U] u_{\mathrm{b}}^{\star}, } \\
p= & \rho g[D]\left(D^{\star}-z^{\star}\right)-2[\tau] \epsilon / \nu \frac{\partial U^{\star}}{\partial X^{\star}}+[\tau] p^{\star},
\end{aligned}
$$

where $\mathbf{i}$ and $\mathbf{k}$ are the $x$ - and $z$-unit vectors, respectively. The decompositions of $\mathbf{u}$ and $p$ introduced here may seem overelaborate at first sight; they do, however, lead to some convenient simplifications later.

For a given ice-stream geometry, one can estimate the thickness and length scales $[D]$ and $[L]$ and the roughness scale $[h]$. It remains to define the other scales in terms of these. Clearly, the driving stress is

$$
\left[\tau_{\mathrm{d}}\right]=\rho g[D] \epsilon .
$$

If the aspect ratio of a typical local bed bump is defined as

$$
\nu \doteq \frac{[h]}{[D]}
$$

then the assumption that form drag in Equation (1) is a significant term in force balance suggests that (cf. Fowler, 1981)

$$
[\tau]=\frac{\left[\tau_{d}\right]}{\nu} .
$$

If basal bumps are shallow with $\nu \ll 1$, as will be assumed, then $[\tau] \gg\left[\tau_{\mathrm{d}}\right]$ and hence deformational velocities will be much greater than any shearing velocities due to the driving stress $\left(u_{\mathrm{S}} \text { defined in section } 3\right)^{1}$. This explains why both components of the local "deformational" part of the velocity field are scaled with $[u]$ in Equation (19), and Equation (9) suggests we put

$$
\frac{[u]}{[U]}=\nu
$$

But $[\tau]$ is a typical deviatoric stress, and so we have

$$
[\tau]=\frac{\eta[u]}{[D]}
$$

Combining Equations (26-28) gives us $[U]$ and $[u]$ :

$$
[u]=\frac{\left[\tau_{\mathrm{d}}\right][D]}{\eta \nu}, \quad[U]=\frac{\left[\tau_{\mathrm{d}}\right][D]}{\eta \nu^{2}}
$$

Note that this derivation assumes that $[U]$ is determined primarily by form drag, and not by the sliding law $\tau_{\mathrm{b}}\left(u_{\mathrm{b}}, x\right)$. In other words, the scaled basal shear stress $\tau_{\mathrm{b}}^{\star} \sim \tau_{\mathrm{b}}([U], x) /\left[\tau_{\mathrm{d}}\right]$ must be $O(1)$ (or small) for all $x$ and the value of $[U]$ calculated here.

In a more realistic model, one might use a Glen's law

\footnotetext{
1 "Shearing velocity" is here used to refer to the velocity which arises if the ice stream flows in simple shear, with the driving stress supported locally at the base and no bed bumps present, while "deformational velocities" refers to non-laminar velocities introduced by the presence of bed topography.
}

rheology $\dot{\varepsilon}=A \tau^{n}$ with an exponent $n$ rather than a fixed $\eta$, and replace Equation $(28)$ by $[\tau]=A^{-1 / n}([u] /[D])^{1 / n}$, from which one obtains

$$
[u]=\frac{A\left[\tau_{\mathrm{d}}\right]^{n}[D]}{\nu^{n}}, \quad[U]=\frac{A\left[\tau_{\mathrm{d}}\right]^{n}[D]}{\nu^{n+1}} .
$$

Using $\left[\tau_{\mathrm{d}}\right]=10^{4} \mathrm{~Pa},[D]=1 \mathrm{~km}, n=3, A=10^{-24} \mathrm{~s}^{-1} \mathrm{~Pa}^{-3}$ and $[h]=50 \mathrm{~m}$ (as a representative drumlin height scale), one obtains $[U] \approx 5 \mathrm{~km} \mathrm{a}^{-1}$, which is clearly about an order of magnitude too large for the Siple Coast ice streams (which have low driving stresses of $\sim 10^{4} \mathrm{~Pa}$ ), suggesting that glacial bedforms are unlikely to generate significant form drag. However, as drumlins usually have steep upstream faces, and the estimate (27) is based on approximating the local bed slope by $\nu$, the value of $\nu=0.05$ may in fact underestimate the effect of bedforms. This is significant because $[U]$ in Equation (30) depends strongly on $\nu$; with the values above but $\nu=0.1$ instead of 0.05 , we find $[U] \approx 315 \mathrm{~m} \mathrm{a}^{-1}$. Moreover, we find in section 7 that even a conservative estimate of $\nu$ can give rise to realistic sliding velocities.

It remains to fix $[d]$, the scale for surface perturbations. Two mechanisms affect how large surface perturbations will be. Firstly, advection in Equation (6) suggests

$$
\frac{[d][U]}{[D]}=[u] \quad \Rightarrow \quad[d]=\nu[D],
$$

and, secondly, hydrostatic pressure changes in Equation (5) would lead to

$$
\rho g[d]=[\tau] \quad \Rightarrow \quad[d]=\frac{\epsilon[D]}{\nu} .
$$

For a consistent model, one should choose the smaller choice of $[d]$ above. For the present we choose Equation (31), and consider later in section 5.2 the rescaling required when $\epsilon / \nu \ll \nu$.

\section{MULTIPLE-SGALES EXPANSION}

The scaled variables defined in the previous section are substituted in the model in section 2, and we omit the asterisks for convenience. All dependent variables other than $H, D$ and $U$ are assumed to depend on both inner and outer variables, which we treat as independent. In order that ordering in a multiple-scales expansion be preserved uniformly with respect to the inner variables, dependent variables must be bounded functions of the inner variables, $x$ and $t$ in our case. Furthermore, the averaging procedures (15-17) now yield

$$
\begin{gathered}
\lim _{R \rightarrow \infty} \frac{1}{2 R} \int_{-R}^{R} h(x, X) \mathrm{d} x=0, \\
\lim _{R \rightarrow \infty} \frac{1}{2 R} \int_{-R}^{R} d(x, t, X, T) \mathrm{d} x=0, \\
\left.\lim _{R \rightarrow \infty} \frac{1}{2 R} \int_{-R}^{R} u(x, t, X, T)\right|_{z=H+\nu h} \mathrm{~d} x=0 .
\end{gathered}
$$

The dimensionless model contains two small parameters, $\nu$ and $\epsilon$. We shall assume that $\epsilon \ll \nu \ll 1$, and construct a leading-order model on this basis. The assumption that $\epsilon \ll \nu$ is clearly realistic for most glacial bedforms; moreover, it ensures that deviatoric stresses $[\tau]=\rho g[D] \epsilon / \nu$ are much smaller than hydrostatic pressures $\rho g[D]$. If this were not the case, one would expect widespread cavitation even in the absence of pressurized subglacial water.

As the boundaries of the ice-flow domain are 
$z=H+\nu h$ and $z=D+\nu d$ and $\nu \ll 1$, the boundary conditions are expanded in Taylor series about $z=H$ and $z=D$ under the assumption that the flow field is sufficiently smooth to allow expansion to the required order. This approach can also be found in Nye (1969) and Morland (1976a, b), and allows the problem to be considered on the simpler domain $H<z<D$.

\subsection{Expansion and simplification}

To the error indicated, the scaled field equations become

$$
\begin{gathered}
\nabla^{2} \mathbf{u}-\nabla p-\mathbf{i} \nu \frac{\partial D}{\partial X}=O\left(\epsilon^{2} / \nu\right), \\
\nabla \cdot \mathbf{u}=\frac{\partial u}{\partial x}+\frac{\partial w}{\partial z}=0,
\end{gathered}
$$

while boundary conditions (4) and (5) at the surface are

$$
\begin{aligned}
& \frac{\partial u}{\partial z}+\frac{\partial w}{\partial x}+\nu\left[d\left(\frac{\partial^{2} u}{\partial z^{2}}+\frac{\partial^{2} w}{\partial x \partial z}\right)+2 \frac{\partial d}{\partial x}\left(\frac{\partial w}{\partial z}-\frac{\partial u}{\partial x}\right)\right] \\
& =O\left(\epsilon, \nu^{2}\right) \quad \text { on } z=D, \\
& p-2 \frac{\partial w}{\partial z}=\frac{\nu^{2}}{\epsilon} d+O(\nu) \quad \text { on } z=D .
\end{aligned}
$$

In Equation (6) $\left.u\right|_{z=D+\nu d}$ and $\left.w\right|_{z=D+\nu d}$ are expanded in Taylor series to some order $n$ about $z=D$

$$
\begin{aligned}
\frac{1}{\nu} \frac{\partial D}{\partial t} & +\frac{\partial d}{\partial t}+U \frac{\partial d}{\partial x}+\sum_{r=1}^{n} \frac{\nu^{r} d^{r-1}}{(r-1) !} \frac{\partial^{r-1} u}{\partial z^{r-1}} \frac{\partial d}{\partial x} \\
& +\frac{\epsilon}{\nu}\left(\frac{\partial D}{\partial T}+U \frac{\partial D}{\partial X}\right)=w+\sum_{r=1}^{n} \frac{\nu^{r} d^{r}}{r !} \frac{\partial^{r} w}{\partial z^{r}} \\
& +\frac{\epsilon}{\nu}\left(U \frac{\partial H}{\partial X}-(D-H) \frac{\partial U}{\partial X}\right) \\
& +O\left(\epsilon, \nu^{n+1}\right) \quad \text { on } z=D,
\end{aligned}
$$

and at the base:

$$
\begin{array}{rc}
\frac{\partial u}{\partial z}+\frac{\partial w}{\partial x}+\nu\left[h\left(\frac{\partial^{2} u}{\partial z^{2}}+\frac{\partial^{2} w}{\partial x \partial z}\right)+2 \frac{\partial h}{\partial x}\left(\frac{\partial w}{\partial z}-\frac{\partial u}{\partial x}\right)\right] \\
=\nu \tau_{\mathrm{b}}+O\left(\epsilon, \nu^{2}\right) & \text { on } z=H, \\
\tau_{\mathrm{b}}=\tau_{\mathrm{b}}(U, x, X)+O(\nu) & \text { on } z=H .
\end{array}
$$

In Equation (9), we expand in a similar manner to Equation (40) above,

$$
\begin{aligned}
& w+\sum_{r=1}^{n} \frac{\nu^{r} h^{r}}{r !} \frac{\partial^{r} w}{\partial z^{r}} \\
& =U \frac{\partial h}{\partial x}+\sum_{r=1}^{n} \frac{\nu^{r} h^{r-1}}{(r-1) !} \frac{\partial^{r-1} u}{\partial z^{r-1}} \frac{\partial h}{\partial x}+O\left(\epsilon, \nu^{n+1}\right) .
\end{aligned}
$$

Equations (40) and (43) are simplified by introducing an averaging operator as

$$
\langle f\rangle=\lim _{R \rightarrow \infty} \frac{1}{2 R} \int_{-R}^{R} f(x, t, z, X, T) \mathrm{d} x
$$

for any $f$ for which $\langle f\rangle$ is well defined. Note that $\langle F\rangle=F$ for any $F$ independent of $x$ (essentially the smoothed quantities $U, D$ and $H$ ), and from Equations (33) and (34), $\langle d\rangle=\langle h\rangle=0$. Moreover, if $f$ is bounded with respect to $x$, then

$$
\left\langle\frac{\partial f}{\partial x}\right\rangle=\lim _{R \rightarrow \infty} \frac{[f]_{x=-R}^{x=R}}{2 R}=0
$$

where $[f]_{x=a}^{x=b}=f(b)-f(a)$ in the usual notation. Armed with these properties of $\langle\cdot\rangle$, we are now ready to manipulate Equations (40) and (43). Now, from Equation (43)

$$
\begin{gathered}
\langle w\rangle+\sum_{r=1}^{n} \frac{\nu^{r}}{r !}\left\langle h^{r} \frac{\partial^{r} w}{\partial z^{r}}\right\rangle \\
=\left\langle U \frac{\partial h}{\partial x}\right\rangle+\sum_{r=1}^{n} \frac{\nu^{r}}{(r-1) !}\left\langle\frac{\partial^{r-1} u}{\partial z^{r-1}} h^{r-1} \frac{\partial h}{\partial x}\right\rangle+O\left(\nu^{n+1}, \epsilon\right) \\
\text { on } z=H .
\end{gathered}
$$

By integration by parts,

$$
\begin{aligned}
& \frac{\nu^{r}}{(r-1) !}\left\langle\frac{\partial^{r-1} u}{\partial z^{r-1}} h^{r-1} \frac{\partial h}{\partial x}\right\rangle=\frac{\nu^{r}}{r !}\left\langle\frac{\partial^{r-1} u}{\partial z^{r-1}} \frac{\partial h^{r}}{\partial x}\right\rangle \\
= & \frac{\nu^{r}}{r !}\left(\lim _{R \rightarrow \infty} \frac{\left[\frac{\partial^{r-1} u}{\partial z^{r-1}} h^{r}\right]_{x=-R}^{x=R}}{2 R}-\left\langle h^{r} \frac{\partial^{r} u}{\partial z^{r-1} \partial x}\right\rangle\right) .
\end{aligned}
$$

The first term on the righthand side vanishes by Equation (45). Therefore, by Equation (37)

$$
\frac{\nu^{r}}{(r-1) !}\left\langle\frac{\partial^{r-1} u}{\partial z^{r-1}} h^{r-1} \frac{\partial h}{\partial x}\right\rangle=\frac{\nu^{r}}{r !}\left\langle h^{r} \frac{\partial^{r} w}{\partial z^{r}}\right\rangle .
$$

Substituting Equation (48) in Equation (46) and using the properties of $\langle\cdot\rangle$ listed above yields

$$
\langle w\rangle=O\left(\nu^{n+1}, \epsilon\right)=O(\epsilon) \quad \text { on } \quad z=H
$$

if, formally, an integer $n$ exists such that $\nu^{n+1} \lesssim \epsilon$, and the expansions in Equations (40) and (43) can be carried out to this order.

Applying $\langle\cdot\rangle$ to both sides of Equation (37) and using Equation (45) yields

$$
\frac{\partial\langle w\rangle}{\partial z}=0
$$

and so $\langle w\rangle=O(\epsilon)$ for all $z$.

In an anologous manner to the derivation of Equation (49), applying $\langle\cdot\rangle$ to both sides of Equation (40) and manipulating yields

$$
\frac{\partial D}{\partial t}+\epsilon\left(\frac{\partial D}{\partial T}+\frac{\partial(D-H) U}{\partial X}\right)=O(\epsilon \nu),
$$

which leads to the conclusion that $D$ depends only on the outer time variable $T$ to $O(\epsilon)$, so $D=D(X, T)$ to $O(\epsilon)$; this is hardly surprising since one would expect the mean thickness of the ice stream to change on the convective time-scale associated with its length (and not its thickness). Moreover, if we define $\bar{U}$ as an average in time analogous to the spatial average $\langle\cdot\rangle$, then Equation (51) reduces to the familiar plugflow mass conservation equation for $D$,

$$
\frac{\partial D}{\partial T}+\frac{\partial(D-H) \bar{U}}{\partial X}=O(\nu)
$$

Equation (51) substituted back in Equation (40) yields the kinematic boundary condition

$$
\frac{\partial d}{\partial t}+U \frac{\partial d}{\partial x}=w+O(\nu) \quad \text { on } z=D .
$$

\subsection{Leading-order model}

Dependent variables are expanded as $\mathbf{u}=\mathbf{u}_{0}+O(\nu)$, $d=d_{0}+O(\nu)$, etc. This yields the following leading-order (to an $O(\nu)$ error) model. 
From Equations (36) and (37),

$$
\begin{gathered}
\nabla^{2} \mathbf{u}_{0}-\nabla p_{0}=0, \\
\nabla \cdot \mathbf{u}_{0}=0,
\end{gathered}
$$

on the domain $H<z<D_{0}$, which is a semi-infinite strip with respect to the inner coordinates, as $H$ and $D$ only depend on the outer ones, $X$ and $T$, to leading order. Note that, to leading order, we have a non-shearing flow; this is again analogous to classical sliding with shallow bed slopes (e.g. Fowler, 1986), and arises because $[\tau] \gg\left[\tau_{\mathrm{d}}\right]$.

From Equations (38) and (39), boundary conditions are

$$
\begin{gathered}
\frac{\partial u_{0}}{\partial z}+\frac{\partial w_{0}}{\partial x}=0 \quad \text { on } z=D_{0}, \\
p_{0}-2 \frac{\partial w_{0}}{\partial z}=\frac{\nu^{2}}{\epsilon} d_{0} \quad \text { on } z=D_{0},
\end{gathered}
$$

and from Equation (52)

$$
\frac{\partial d_{0}}{\partial t}+U_{0} \frac{\partial d_{0}}{\partial x}=w_{0} \quad \text { on } z=D_{0},
$$

while from Equations (41) and (43) one obtains

$$
\begin{array}{cc}
\frac{\partial u_{0}}{\partial z}+\frac{\partial w_{0}}{\partial x}=0 & \text { on } z=H, \\
w_{0}=U_{0} \frac{\partial h}{\partial x} & \text { on } z=H .
\end{array}
$$

Note that, because the flow is non-shearing and $[\tau] \gg\left[\tau_{\mathrm{d}}\right]$, there is zero basal shear stress at leading order in Equation $(58) ; \tau_{\mathrm{b}}$ in Equation (38) is a $O(\nu)$ correction. We should also comment at this point about the $O\left(\nu^{2} / \epsilon\right)$ term in Equations (39) and (56); its retention as a possible $O(1)$ term is justified since, by virtue of $\epsilon \ll \nu$, we have $\nu^{2} / \epsilon \gg \nu$. Hence the $O\left(\nu^{2} / \epsilon\right)$ term is much greater than the $O(\nu)$ error in the leading-order model. We wish to retain the term explicitly because, as will be seen in the next section, it is responsible for the relaxation of surface perturbations to their steadystate shape. There remains the possibility that $\nu^{2} / \epsilon \gg 1$, in which case a rescaling becomes necessary. This rescaling, $d^{\star \star}=\left(\nu^{2} / \epsilon\right) d^{\star}$, introduces no new terms into the leadingorder model, and the error remains of $O(\nu)$. The rescaling converts Equations (56) and (57), respectively, into (again dropping asterisks)

$$
\begin{gathered}
p_{0}-2 \frac{\partial w_{0}}{\partial z}=d_{0} \quad \text { on } z=D_{0} \\
\frac{\epsilon}{\nu^{2}}\left(\frac{\partial d_{0}}{\partial t}+U_{0} \frac{\partial d_{0}}{\partial x}\right)=w_{0} \quad \text { on } z=D_{0} .
\end{gathered}
$$

One may then wish to ignore the $O\left(\epsilon / \nu^{2}\right)$ terms on the lefthand side in Equation (61). This is a singular perturbation, as it ignores the time derivative in $d$, and given initial conditions then require a boundary layer in $t$. When such a boundary layer is taken into account, the behaviour of the original leading-order model and its rescaled leading-order counterpart then differs by a (small) term of $\left(\epsilon / \nu^{2}\right) U_{0} \partial d_{0} / \partial x$, and one may therefore persevere with the original leading-order model even when $\nu^{2} / \epsilon \gg 1$.

Given $D_{0}$ and $H$ (which are constant with respect to the inner variables $x$ and $t$ ), Equations (53-59) almost constitute a closed set of equations (given appropriate initial conditions); it remains to fix the sliding velocity $U_{0}$ (independent of the inner spatial variable $x$, but dependent on time $t$ ), which is in fact the object of our study. Simple considerations of momentum conservation suggest that one should expect, by analogy with Nye (1969) and Fowler (1981), a leadingorder relation of the form

$$
\begin{aligned}
-\left(D_{0}-H\right) \frac{\partial D_{0}}{\partial X} & =\left\langle\left.\left(p_{0}-2 \frac{\partial w_{0}}{\partial z}\right)\right|_{z=H} \frac{\partial h}{\partial x}\right\rangle \\
& +\left\langle\tau_{\mathrm{b}}\left(U_{0}, x, X\right)\right\rangle,
\end{aligned}
$$

where the term on the left is the mean driving stress, and the terms on the right are the mean component of normal stress in the upstream direction and the mean basal shear stress, respectively. It can, in fact, be shown in a rather convoluted manner, by considering $O(\nu)$ terms in Equations (36-38), (41) and (42) in the multiple-scales expansion, that Equation (62) is indeed correct. This equation finally determines $U_{0}$.

Equation (62) relates mean driving stress to mean sliding velocity; this is very different from shallow-ice theory where local driving stress determines local sliding velocity. This may be understood as an extreme example of the effect of longitudinal stresses considered in, for example, Kamb and Echelmeyer (1986). Since the ice is stiff here in the sense that deformational velocities $[u]$ are much less than sliding velocities $[U]$, longitudinal stresses arise which ensure that the sliding velocity is locally constant in space to leading order. In particular, if $\tau_{\mathrm{b}} \neq 0$, a sliding velocity independent of position requires basal shear stress to be concentrated where the bed is stickier (i.e. where $\tau_{\mathrm{b}}$ is greater).

\section{SOLUTION OF THE LOGAL FLOW PROBLEM}

One of the main benefits of the results of the previous section is that the free boundary problem for the ice-stream surface $D$ is reduced to a fixed boundary problem on the "inner" scale; the surface perturbation $d_{0}$ appears as a variable in the boundary conditions of the ice-flow problem, but does not affect the position of the boundary $z=D_{0}$ at leading order. The domain of the leading-order "inner" ice-flow problem is the strip $H<z<D_{0}$.

We will now concern ourselves exclusively with the inner problem at leading order, and therefore omit the subscripts 0 as well as the outer variables $(X, T)$. Furthermore, since $D_{0}$ and $H$ are independent of the inner variables, an appropriate choice of origin and of the scales $[D]$ and $[L]$ ensures that the following hold for any given fixed $(X, T)$ :

$$
H=0, \quad D_{0}=1, \quad \frac{\partial D_{0}}{\partial X}=-1 .
$$

The domain of the problem is then $0<z<1$, and the driving stress is $-\left(D_{0}-H\right)\left(\partial D_{0} / \partial X\right)=1$.

A partial solution of the inner problem can be derived in Fourier transform space. To facilitate matters, we assume that the bed is periodic with period $a$ and define the Fourier transform of a periodic function $f$ as

$$
\begin{aligned}
\hat{f}_{n}(z, t) & =\int_{0}^{a} f(x, z, t) \exp \left(-i k_{n} x\right) / a \mathrm{~d} x \\
k_{n} & =\frac{2 n \pi}{a} \quad n \in Z
\end{aligned}
$$

Note that $n$ here denotes an integer index and not the expo- 


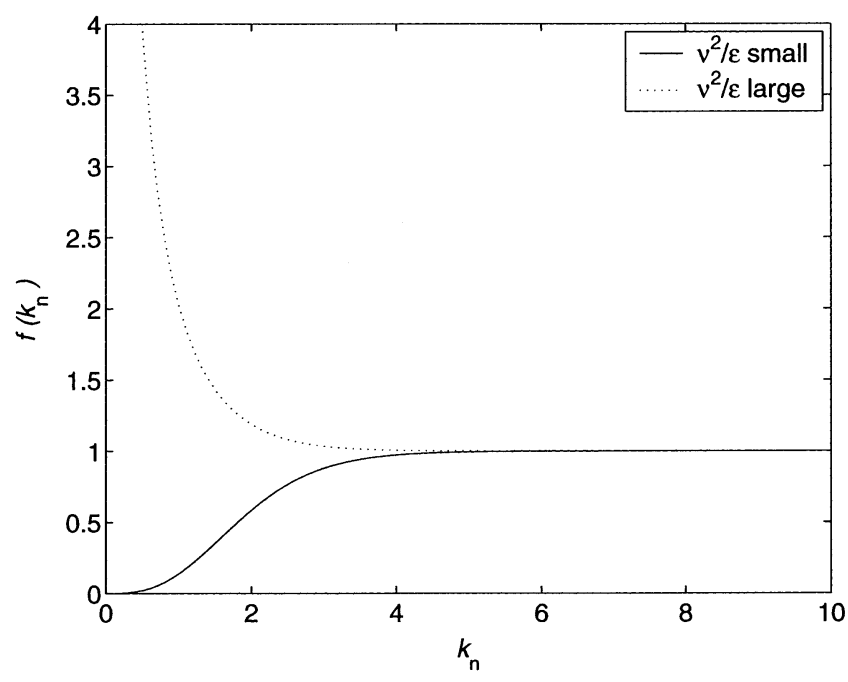

Fig. 4. Form-drag enhancement for $\nu^{2} \ll \epsilon, \nu^{2} \gg \epsilon$, compared with the assumption of infinite depth. Shown here is the function $f\left(k_{n}\right)$ defined in Equation (67).

nent in Glen's law. The evolution of perturbations $d$ on the ice-stream surface is described by

$$
\begin{aligned}
\left(\frac{\partial}{\partial t}+\right. & \left.i k_{n} U+\frac{\nu^{2}}{\epsilon} \frac{\sinh ^{2} k_{n}}{2 k_{n}\left(\sinh k_{n} \cosh k_{n}+k_{n}\right)}\right) \hat{d}_{n} \\
& =\frac{k_{n} \cosh k_{n}+\sinh k_{n}}{\sinh k_{n} \cosh k_{n}+k_{n}} i k_{n} U \hat{h}_{n},
\end{aligned}
$$

while momentum conservation (Equation (49), which determines $U(t)$ at any time $t)$ is expressed as

$$
\begin{gathered}
1=\sum_{n=1}^{\infty} 4 k_{n}^{3} \frac{\sinh ^{2} k_{n}-k_{n}^{2}}{k_{n}+\cosh k_{n} \sinh k_{n}}\left|\hat{h}_{n}\right|^{2} U \\
+\sum_{n=1}^{\infty} 2 \frac{\nu^{2}}{\epsilon} k_{n} \frac{\sinh k_{n}+k_{n} \cosh k_{n}}{k_{n}+\cosh k_{n} \sinh k_{n}} \Im\left(\hat{d}_{n} \hat{h}_{n}^{*}\right)+\left\langle\tau_{\mathrm{b}}(U, x)\right\rangle,
\end{gathered}
$$

where $^{*}$ denotes complex conjugation and $\Im$ stands for imaginary part.

The evolution equation (63) for $d$ is not dissimilar to some of Gudmundsson and others' (1998) results. The first two terms on the lefthand side are growth and advection-type terms, while the third leads to the decay of surface perturbations due to increased hydrostatic pressure. The term on the righthand side is a source term which represents how well basal perturbations are "transmitted" to the surface. A notable departure from Gudmundsson's results is that the presence of small-scale roughness $\tau_{\mathrm{b}}$ does not affect surface evolution at leading order in our model, though it will be important at higher order. Moreover, Equation (63) is not a linear equation as $U$ depends on the $\hat{d}_{n}$ through Equation (64). A simple analytical solution of the form presented in Gudmundsson and others (1998) is therefore not available.

For a bed which can be represented by a truncated Fourier series, it is not difficult to solve Equations (63) and (64) numerically for appropriate initial conditions on $d$ (cf. section 7). Here we focus on qualitative features of Equation (64). In particular, approximate versions of Equation (64) which depend only on the $\hat{h}_{n}$ can be derived in the case of $\nu^{2} \gg \epsilon$ and $\nu^{2} \ll \epsilon$.

When $\nu^{2} \ll \epsilon$ (small bed roughness or large driving stress), one simply ignores the $O\left(\nu^{2} / \epsilon\right)$ term in Equation (64) and obtains

$$
1=\sum_{n=1}^{\infty} 4 k_{n}^{3} \frac{\sinh ^{2} k_{n}-k_{n}^{2}}{k_{n}+\cosh k_{n} \sinh k_{n}}\left|\hat{h}_{n}\right|^{2} U+\left\langle\tau_{\mathrm{b}}\right\rangle .
$$

In the case $\nu^{2} \gg \epsilon$ (large bed roughness or small driving stress) one rescales $d^{\star \star}=\left(\nu^{2} / \epsilon\right) d^{\star}$ (cf. section 5.2). Ignoring the advection and growth terms of $O\left(\epsilon / \nu^{2}\right)$ in the rescaled version of Equation (63) gives $\hat{d}_{n}$ in terms of $\hat{h}_{n}$; substituting this in Equation (64) yields

$$
1=\sum_{n=1}^{\infty} 4 k_{n}^{3} \frac{k_{n}+\sinh k_{n} \cosh k_{n}}{\sinh ^{2} k_{n}}\left|\hat{h}_{n}\right|^{2} U+\left\langle\tau_{\mathrm{b}}\right\rangle .
$$

Ignoring the friction term $\left\langle\tau_{\mathrm{b}}\right\rangle$, both of these are of the form

$$
1=\sum_{n=1}^{\infty} 4 k_{n}^{3} f\left(k_{n}\right)\left|\hat{h}_{n}\right|^{2} U
$$

Classical (infinite-depth) sliding theory predicts $f\left(k_{n}\right)=1$ (e.g. Fowler, 1986, equation (2.38)). Thus $f\left(k_{n}\right)$ measures the enhancement of basal drag due to surface effects compared with the classical result. Figure 4 shows $f\left(k_{n}\right)$ for the two cases considered above. We see that $f\left(k_{n}\right) \rightarrow 1$ for large $k_{n}$ as required. However, at small $k_{n}$ (i.e. large wavelengths, $\lambda=2 \pi / k_{n}$ ) we see that form drag is suppressed for higher driving stresses or lower bed roughnesses compared with the classical result, whereas form drag is enhanced for smaller driving stresses or large bed roughnesses by the formation of a standing wave at the ice-stream surface. These results are also confirmed by direct numerical simulation of Equations (63) and (64).

Note that $1 / f\left(k_{n}\right)$ is essentially Gudmundsson's (1997) "sliding function" $s$ evaluated for a Glen's law exponent $n=1$ at small bed slope ( $\varepsilon=0$ in his notation) but at finite "thinness parameter" ( $\delta$ in his paper, here simply $\left.1 / k_{n}\right)$, while Gudmundsson dealt only with the case $\delta \ll 1$. Our results suggest a major discrepancy with Gudmundsson's equation (36), which suggests $s$ should be independent of "thinness" $\delta$, or $k_{n}$ here, when bed roughness is small. Moreover, the different behaviour in the limits $\nu^{2} / \epsilon \rightarrow 0, \infty$ suggests that $s$ should also depend on ice surface slope for finite $\delta$.

\section{NUMERICAL SOLUTION}

We deliberately chose the simplest possible rheology for ice in section 2 in order to simplify our model derivation. However, a typical ice stream is polythermal, and temperature variations with depth will affect its rheology (Paterson, 1994). This, in turn, is likely to affect both form drag and surface perturbations caused by large obstacles.

The simplest assumption one can make is that viscosity increases exponentially with height above the bed, $\eta \sim \exp (\kappa z)$ (Gudmundsson and others, 1998), which can be justified theoretically if temperature varies linearly with depth and the dependence of viscosity on temperature is described by a Frank-Kamenetskii approximation (e.g. Fowler, 1997, p. 184). 


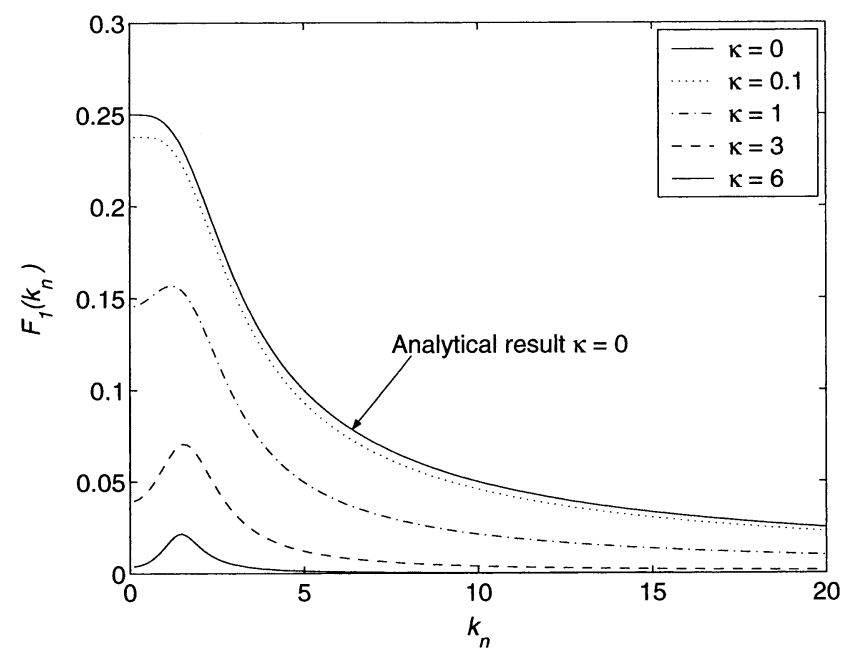

Fig. 5. The function $F_{1}\left(k_{n}, \kappa\right)$ at various values of $\kappa_{\text {. Note that }}$ $F_{1}$ determines how effectively a given Fourier mode will decay in Equation (75). Clearly $F_{1}$ decreases with $\kappa$. Decay of surface perturbations is suppressed by stiffer ice near the surface.

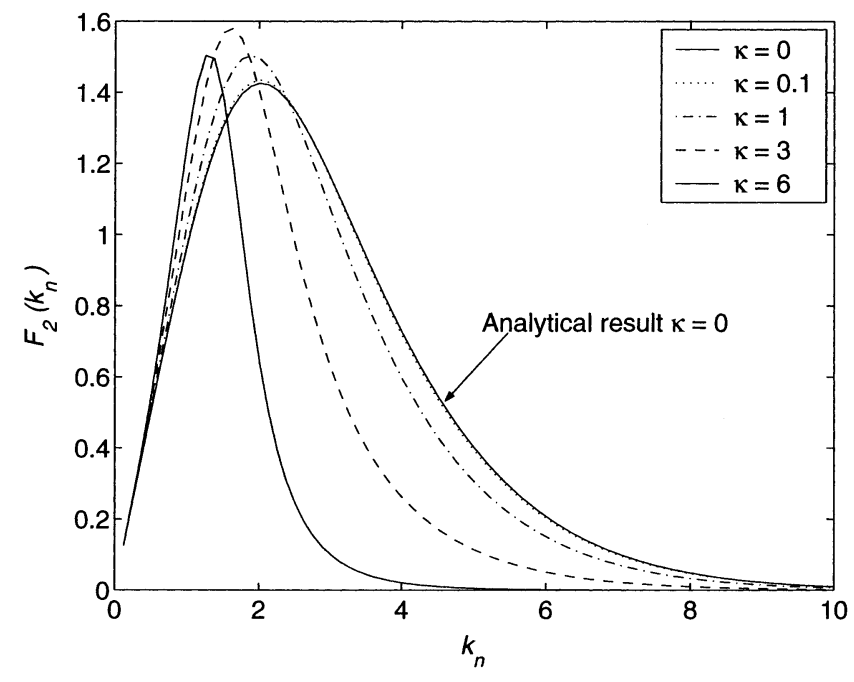

Fig. 6. The function $F_{2}\left(k_{n}, \kappa\right)$ at various values of $\kappa$. Note that $F_{2}$ controls how well basal perturbations are "transmitted" to the surface; with increasing $\kappa$ the band of effectively transmitted wavelengths becomes narrower and shifted towards larger wavelengths $\lambda=2 \pi / k_{n}$.

The appropriately modified "inner" problem becomes $\left(\kappa \frac{\partial \mathbf{u}}{\partial z}+\kappa \nabla w+\nabla^{2} \mathbf{u}\right) \exp (\kappa z)-\nabla p=0 \quad$ on $z \in(0,1)$

$$
\begin{gathered}
\nabla \cdot \mathbf{u}=0 \quad \text { on } z \in(0,1), \\
\frac{\partial u}{\partial z}+\frac{\partial w}{\partial x}=0 \quad \text { on } z=0, \\
w=U \frac{\partial h}{\partial x} \quad \text { on } z=0, \\
\frac{\partial u}{\partial z}+\frac{\partial w}{\partial x}=0 \quad \text { on } z=1, \\
p-2 \exp \kappa \frac{\partial w}{\partial z}=\frac{\nu^{2}}{\epsilon} d \quad \text { on } z=1, \\
\frac{\partial d}{\partial t}+U \frac{\partial d}{\partial x}=w \quad \text { on } z=1,
\end{gathered}
$$

while Equation (49) still holds.

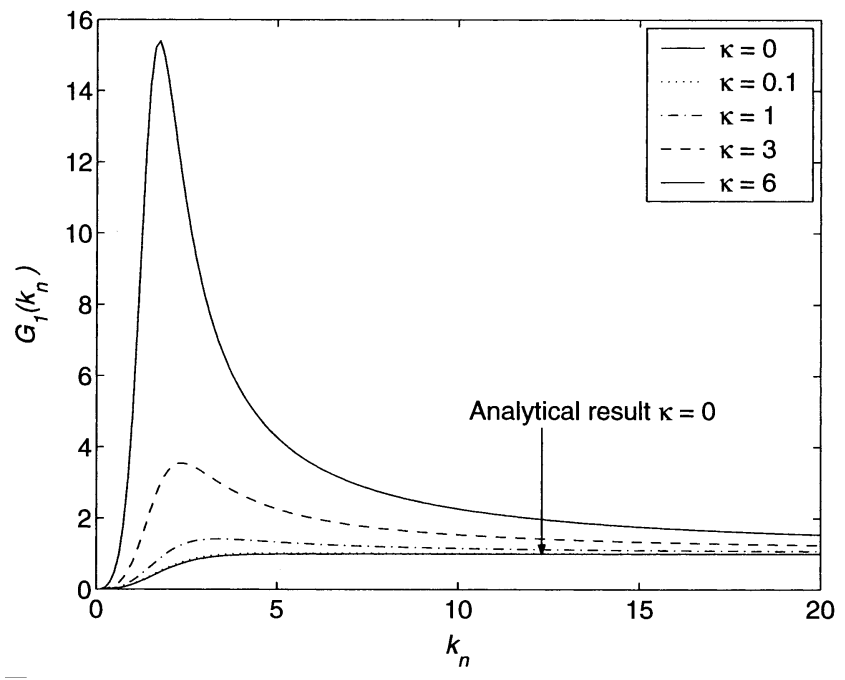

Fig. 7. The function $G_{1}\left(k_{n}, \kappa\right)$ at various values of $\kappa$. Note that $G_{1}$ determines how form drag is enhanced compared with the classical (infinite-depth) result in the first momentum balance term in Equation (76). $G_{1}$ develops a pronounced peak at $k_{n} \approx 2$ for large $\kappa$. One may ascribe this to flow over bumps of this wavenumber involving more deformation of the upper, stiffer ice, leading to increased resistance.

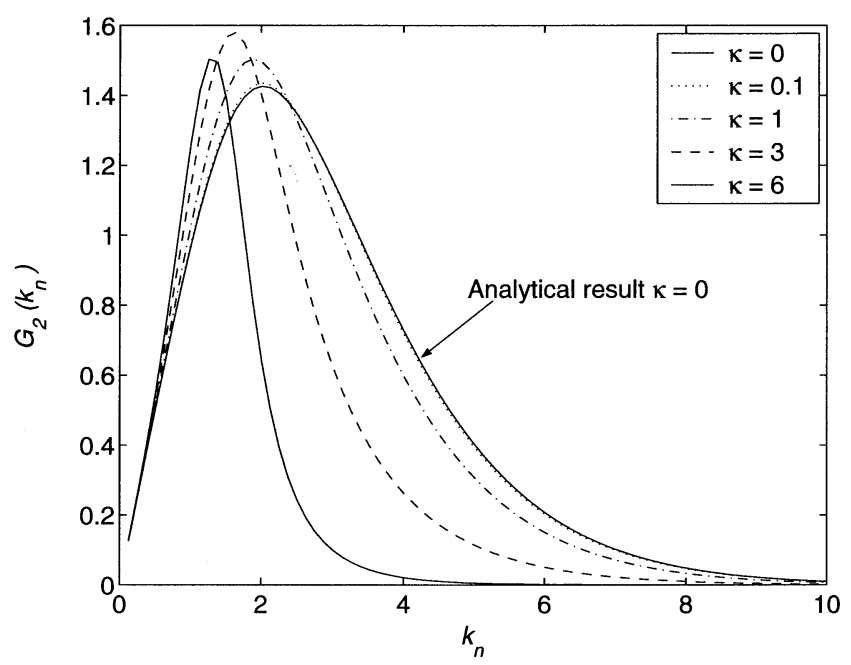

Fig. 8. The function $G_{2}\left(k_{n}, \kappa\right)$ at various values of $\kappa$.

A partial solution in Fourier transform space is again possible, with analogues to Equations (63) and (64):

$$
\begin{aligned}
\left(\frac{\partial}{\partial t}+\right. & \left.i k_{n} U+\frac{\nu^{2}}{\epsilon} F_{1}\left(k_{n}, \kappa\right)\right) \hat{d}_{n}=i F_{2}\left(k_{n}, \kappa\right) U \hat{h}_{n}, \\
1= & \sum_{n=1}^{\infty} 4 k_{n}^{3} G_{1}\left(k_{n}, \kappa\right)\left|\hat{h}_{n}\right|^{2} U \\
& +\sum_{n=1}^{\infty} 2 \frac{\nu^{2}}{\epsilon} G_{2}\left(k_{n}, \kappa\right) \Im\left(\hat{d}_{n} \hat{h}_{n}^{*}\right)+\left\langle\tau_{\mathrm{b}}\right\rangle
\end{aligned}
$$

where the various functions $F$ and $G$ have to be calculated numerically (cf. Appendix). Figures 5-8 show numerical calculations of the functions $F$ and $G$ for various values of $\kappa$. Reasonable estimates for $\kappa$ are $\kappa \approx 3-6$, using (cf. Paterson, 1994, p. 86)

$$
\eta \propto \exp (Q / R T) \approx \exp \left[Q / R T_{0}-Q\left(T-T_{0}\right) / R T_{0}^{2}\right],
$$

with $R=8.3 \mathrm{~J} \mathrm{~mol}^{-1} \mathrm{~K}^{-1}, Q=0.7-1.4 \times 10^{5} \mathrm{~J} \mathrm{~mol}^{-1}, T_{0}=270 \mathrm{~K}$ and a temperature difference between surface and base of the ice stream of $T-T_{0} \approx-25 \mathrm{~K}$.

Equations (75) and (76) are solved numerically for a 

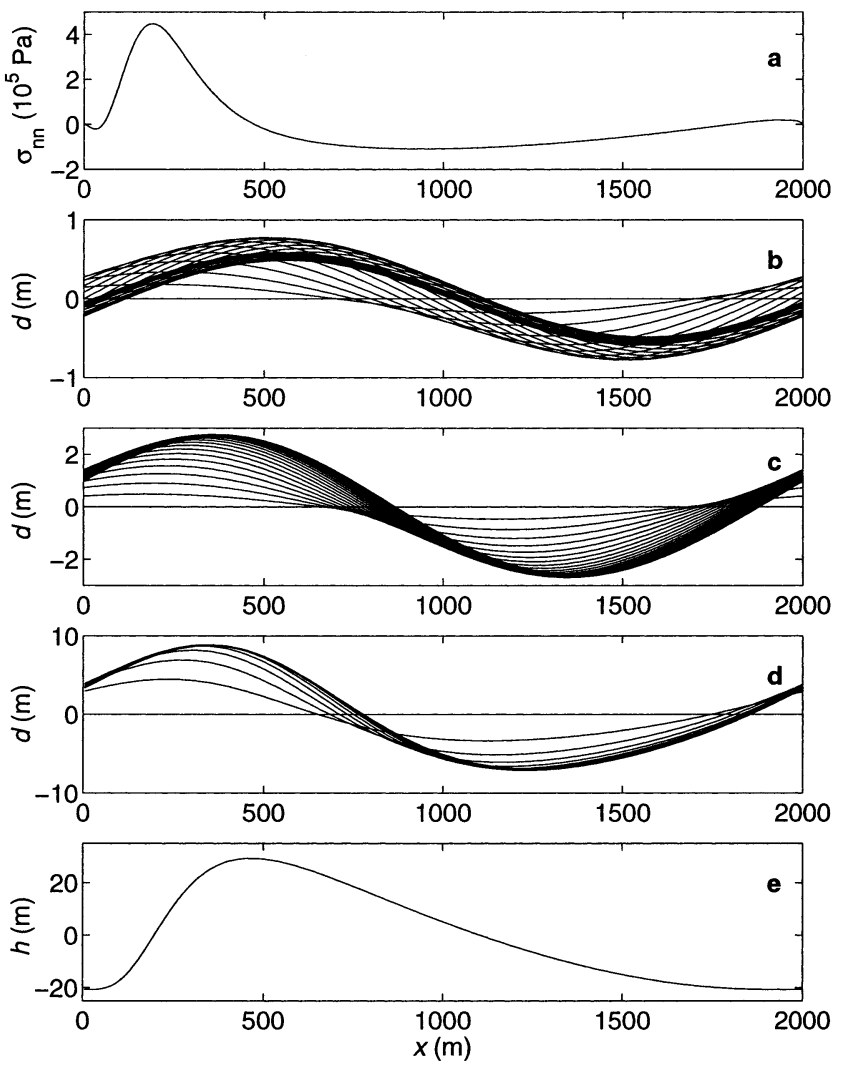

Fig. 9. Simulation of the evolution of surface perturbations caused by the bedform shown in (e) (extended periodically). (b) shows the evolving surface at 120 day intervals for $\kappa=6$, corresponding to an activation energy $Q=1.4 \times 10^{5} \mathrm{f} \mathrm{mol}^{-1}$ and a temperature gradient of about $25 \mathrm{~K}$. (c) shows surface evolution at 24 day intervals for $\kappa=3$, corresponding to an activation energy $Q=7 \times 10^{4} \mathrm{~J} \mathrm{~mol}^{-1}$ and a temperature gradient of about $25 \mathrm{~K}$. (d) shows surface evolution at 12 day intervals for $\kappa=0$, corresponding to the isothermal case. Note the different $y$-axis scales in the different panels. (a) shows the normal stress distribution (minus hydrostatic contribution) at the end of the simulation shown in (b).

given bed and value of $\kappa$, subject to the initial condition $d=0$ and under the assumption $\tau_{\mathrm{b}}=0$. For ease of interpretation, our results are re-dimensionalized. In order to do so (cf. Equation (29)), the ice viscosity $\eta$ has to be estimated at the base of the ice stream, which is tantamount to estimating the effect of the non-linearity of the rheology of ice on our results. Given a bed roughness estimate $\nu$, viscosity is estimated using Glen's law as

$$
\eta=A^{-1}[\tau]^{1-n}=A^{-1}\left[\tau_{\mathrm{b}}\right]^{1-n} \nu^{n-1}
$$

with $A=6 \times 10^{-24} \mathrm{~s}^{-1} \mathrm{~Pa}^{-3}$ and $n=3$. Since $\eta$ above depends strongly on $\nu$, this is a crude way of proceeding, and one should therefore regard our results as entirely qualitative.

The bed chosen for our simulations is shown in Figure 9e, where ice flow here is from left to right. The chosen profile has the steep upstream side typical of a drumlin. The bed is extended periodically, with period $a=2000 \mathrm{~m}$, while ice thickness is put at $1000 \mathrm{~m}$ and the driving stress at $\left[\tau_{\mathrm{d}}\right]=$ $10^{4} \mathrm{~Pa}$, corresponding to $\epsilon \approx 10^{-3}$. The profile has a maximum height differential of $50 \mathrm{~m}$, so we estimate $\nu=0.05$. This yields an approximate viscosity of $\eta=4.2 \times 10^{12} \mathrm{~Pa} \mathrm{~s} \approx 1.3$ bar a.

The results of the simulations are shown in Figures 9 and 10 . For all three values of $\kappa$ used, the surface perturbations $d$ eventually form a standing wave above the bed

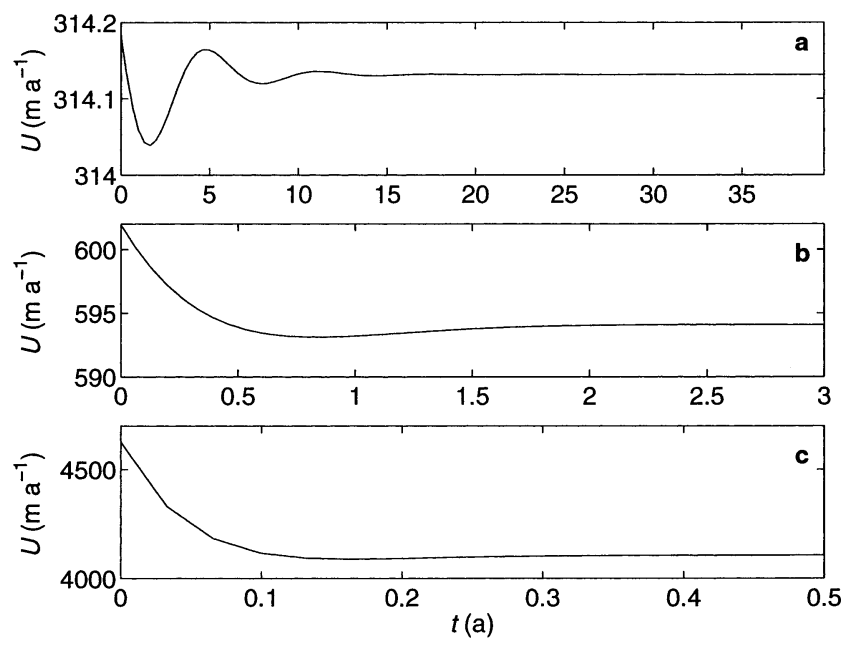

Fig. 10. Evolution of the sliding velocity during the simulations shown in Fig. 9. (a) corresponds to the case $\kappa=6$, (b) to $\kappa=3$ and (c) to $\kappa=0$. Note the different $x$ - and $y$-axis scales in the different panels.

bump, and consequently the sliding velocity approaches a constant at large times. The time taken to approach this steady state depends on $\kappa$; the larger $\kappa$, the more slowly the steady state is attained. This may be attributed to the fact that the function $F_{1}\left(k_{n}, \kappa\right)$ decreases with $\kappa$ (Fig. 5). Inspection of Equation (75) shows that $F_{1}$ controls the "decay" of surface perturbations.

The final sliding velocity is considerably smaller for large $\kappa$ than for small $\kappa$, which indicates that the upper parts of the ice stream, which are stiffer for larger $\kappa$, impede sliding. Furthermore, despite a large estimate for $[U]$ (cf. section 4), realistic values of sliding velocities can be produced.

The size of the standing wave also decreases with $\kappa$. This may be attributed to the fact that the function $F_{2}\left(k_{n}, \kappa\right)$, which controls how well particular wavelengths are transmitted to the surface, decreases with $\kappa$ for $k_{n}>\pi$ (corresponding to wavelengths smaller than two ice thicknesses; cf. Fig. 6). This reduces the size of surface perturbations in the present case, where we have chosen a bedform wavelength of two ice thicknesses.

\section{DISGUSSION}

Our results suggest that long-wavelength $(\sim 1 \mathrm{~km})$ bedforms of sufficient amplitude may be able to limit the sliding velocity of ice streams, particularly if there is a significant temperature gradient in the ice, leading to high viscosities in the near-surface parts of the ice stream.

This conclusion is, however, not entirely robust; the main difficulty to resolve is the effect of the non-linearity of ice rheology - our estimate of $[U]$ in Equation (30) is highly sensitive to $\nu$. Moreover, we have entirely ignored the possibility of cavitation here. Given the typically high drainage pressures under an ice stream, one may suspect that cavitation in the lee of large bedforms is a very real possibility. Figure 9a shows the normal stress distribution (minus hydrostatic pressure) over the bed at the end of the simulation shown in Figure 9b. As expected, normal stress is lowest in the lee of the bedform. A corollary of this is that care needs to be exercised in interpreting observations of water levels in boreholes (e.g. Engelhardt and Kamb, 1997) in order to determine basal effective pressures, as deviatoric 
normal stresses due to flow over basal undulations may affect normal stress and hence basal effective pressure. This effect has previously been considered, albeit in a crude way and in a different context, by Booth and Hallet (1993).

In agreement with Gudmundsson and others' (1998) results, our analysis shows that basal perturbations of wavelengths comparable to ice thickness cause a surface expression at the top of the ice stream. How well a given wavelength is "transmitted" to the surface does, however, depend on the temperature gradient in the ice (and hence on $\kappa$; cf. Fig. 6). For relatively high temperature gradients we may expect to see only weak surface expressions caused by basal bumps of wavelengths of 1-2 ice thicknesses (cf. Fig. 9), while longer wavelengths, which are less efficient at causing form drag, are transmitted more strongly (cf. Fig. 6).

The work presented here also indicates how ice dynamics on length scales comparable to ice thickness can be separated in a mathematically consistent way from large-scale ice dynamics, and it is conceivable that the multiple-scales approach used here could be useful in other circumstances where a consideration of local (kilometre-scale) effects is important. This is not limited to the case of significant form drag, and could be useful, for instance, in tracer studies if there are significant bed undulations on a kilometre scale generating a non-laminar velocity field.

\section{ACKNOWLEDGEMENTS}

I would like to thank L. Morland, H. Gudmundsson and J. Meyssonnier (Scientific Editor) for many suggestions which helped greatly to improve the original manuscript. I should also like to thank A. Fowler for supervising this work as part of a D.Phil. thesis. This work was supported financially by the Engineering and Physical Sciences Research Council and by Corpus Christi College, Oxford University.

\section{REFERENCES}

Booth, D. B. and B. Hallet. 1993. Channel networks carved by subglacial water: observations and reconstruction in the eastern Puget Lowland of Washington. Geol. Soc. Am. Bull., 105(5), 671-683.

Brown, N. E., B. Hallet and D. B. Booth. 1987. Rapid soft bed sliding of the Puget glacial lobe. 7. Geophys. Res., 92(B9), 8985-8997.

Engelhardt, H. and B. Kamb. 1997. Basal hydraulic system of a West Antarctic ice stream: constraints from borehole observations. f. Glaciol., 43(144), 207-230.

Engelhardt, H. and B. Kamb. 1998. Basal sliding of Ice Stream B, West Antarctica. 7. Glaciol., 44(147), 223-230.

Fowler, A. C. 1979. A mathematical approach to the theory of glacier sliding. 7. Glaciol., 23 (89), 131-141.

Fowler, A. C. 1981. A theoretical treatment of the sliding of glaciers in the absence of cavitation. Philos. Trans. R. Soc. London, Ser. A, 298(1445), 637-685.

Fowler, A. C. 1986. A sliding law for glaciers of constant viscosity in the presence of subglacial cavitation. Proc. R. Soc. London, Ser. A, 407(1832), 147-170.

Fowler, A. C. 1997. Mathematical models in the applied sciences. Cambridge,
Cambridge University Press. (Cambridge Texts in Applied Mathematics.) Gudmundsson, G. H. 1997. Basal-flow characteristics of a non-linear flow sliding frictionless over strongly undulating bedrock. f. Glaciol., 43(143), 80-89.

Gudmundsson, G. H., C. F. Raymond and R. Bindschadler. 1998. The origin and longevity of flow stripes on Antarctic ice streams. Ann. Glaciol., 27, $145-152$.

Holmes, M. H. 1995. Introduction to perturbation methods. New York, SpringerVerlag. (Texts in Applied Mathematics, Vol. 20.)

Hutter, K. 1983. Theoretical glaciology; material science of ice and the mechanics of glaciers and ice sheets. Dordrecht, etc., D. Reidel Publishing Co.; Tokyo, Terra Scientific Publishing Co.

Hutter, K., F. Legerer and U. Spring. 1981. First-order stresses and deformations in glaciers and ice sheets. F. Glaciol., 27 (96), 227-270.

Kamb, B. and K. A. Echelmeyer. 1986. Stress-gradient coupling in glacier flow: I. Longitudinal averaging of the influence of ice thickness and surface slope. F. Glaciol., 32(111), 267-284.

Morland, L. W. 1976a. Glacier sliding down an inclined wavy bed. F. Glaciol., $\mathbf{1 7}(77), 447-462$.

Morland, L. W. 1976b. Glacier sliding down an inclined wavy bed with friction. f. Glaciol., 17 (77), 463-477.

Morland, L.W. 2000. Steady plane isothermal linearly viscous flow of ice sheets on beds with moderate slope topography. Proc. R. Soc. London, Ser. A, 456, 1711-1739.

Nye, J. F. 1969. A calculation on the sliding of ice over a wavy surface using a Newtonian viscous approximation. Proc. R. Soc. London, Ser. A, 311(1506), 445-467.

Paterson, W. S. B. 1994. The physics of glaciers. Third edition. Oxford, etc., Elsevier. Reeh, N. 1987. Steady-state three-dimensional ice flow over an undulating base: first-order theory with linear ice rheology. F. Glaciol., 33(114), 177-185.

Whillans, I. M. and C. J. van der Veen. 1997. The role of lateral drag in the dynamics of Ice Stream B, Antarctica. F. Glaciol., 43(144), 231-237.

\section{APPENDIX}

\section{GALGULATION OF FUNGTIONS $F_{1}, F_{2}, G_{1}, G_{2}$}

Equation (69) is satisfied by introducing a stream function $\psi$ as

$$
\mathbf{u}=\left(\frac{\partial \psi}{\partial z},-\frac{\partial \psi}{\partial x}\right)
$$

Fourier-mode solutions of Equations (68) and (69) then take the form

$$
\psi=A \exp (i k x+m z)
$$

where substituting $\psi$ in Equation (68) yields a quartic for $m$, $m^{4}+2 \kappa m^{3}+\left(\kappa^{2}-2 k^{2}\right) m^{2}-2 \kappa k^{2} m+k^{2}\left(\kappa^{2}+k^{2}\right)=0$, which has four distinct roots $m_{1}, m_{2}, m_{3}$ and $m_{4}$ when $\kappa \neq$ 0 (in fact, these roots form complex conjugate pairs). A general Fourier-mode solution

$$
\psi=\sum_{j=1}^{4} A_{j} \exp \left(i k x+m_{j} z\right)
$$

is substituted in the boundary conditions (70-73); the solution of the set of linear algebraic equations in the $A_{j}$ which results is used to calculate the $F$ and $G$ functions by substituting in Equations (74) and (49). 